\title{
NRQCD colour-octet expansion vs. LHC quarkonium production: signs of a hierarchy puzzle?
}

\author{
Pietro Faccioli $^{1, \mathrm{a}}$, Carlos Lourenço ${ }^{2, \mathrm{~b}}$ \\ ${ }^{1}$ LIP, Lisbon, Portugal \\ ${ }^{2}$ CERN, Geneva, Switzerland
}

Received: 27 April 2019 / Accepted: 20 May 2019 / Published online: 30 May 2019

(C) The Author(s) 2019

\begin{abstract}
The observation of unpolarized quarkonium production in high energy pp collisions, at mid rapidity, implies a significant violation of the non-relativistic QCD (NRQCD) velocity scaling rules. A precise experimental confirmation of this picture could definitely rule out the current formulation of the factorization expansion. This conclusion relies on current perturbative determinations of the short-distance kinematic factors and may be reverted if improved calculations would modify, in a very specific way, their transverse momentum dependences. That solution would result, however, in a full degeneracy in the presently assumed basis of ${ }^{2 S+1} L_{J}$ Fock states. Therefore, whatever the outcome, improved polarization measurements will challenge and improve our fundamental understanding of quarkonium production.

Quarkonium production is a central case study for the understanding of QCD bound state formation. Non-relativistic QCD (NRQCD) addresses its description in a rigorous way starting from first principles, assuming the factorization of short- and long-distance effects in the limit of small relative velocity $(v)$ of the heavy quark and antiquark $(Q \bar{Q})$ forming the bound state [1]. Under this hypothesis, the $Q \bar{Q}$ state is modelled mathematically as an expansion over Fock states of determined angular momentum and colour properties, $Q \bar{Q}\left({ }^{2 S+1} L_{J}^{[n]}\right)$, with $L=S, P$, etc., $S=0,1$, etc., $J=0,1,2$, etc., and $n=1$ (colour singlet) or 8 (colour octet). The non-perturbative evolution of such preresonance states to the observed quarkonium is described by constant factors (long-distance matrix elements, LDMEs, $\mathcal{L})$, currently not calculable and determined in global fits to sets of measurements, while perturbative calculations fix the kinematics-dependent short-distance $Q \bar{Q}$ production cross sections (short distance coefficients, SDCs, $\mathcal{S}$ ) for each of
\end{abstract}

\footnotetext{
a e-mail: Pietro.Faccioli@cern.ch

b e-mail: carlos.lourenco@cern.ch
}

the considered terms of the expansion, so that the production cross section of the quarkonium state $H$ in pp collisions is

$$
\begin{aligned}
\sigma(\mathrm{pp} \rightarrow H+X)= & \sum_{S, L, n} \mathcal{S}\left(\mathrm{pp} \rightarrow Q \bar{Q}\left[^{2 S+1} L_{J}^{[n]}\right]+X ; \sqrt{s}, M, p_{\mathrm{T}}, y\right) \\
& \times \mathcal{L}\left(Q \bar{Q}\left[^{2 S+1} L_{J}^{[n]}\right] \rightarrow H\right),
\end{aligned}
$$

where $M, p_{\mathrm{T}}$, and $y$ are the mass, transverse momentum, and rapidity of the $Q \bar{Q}$.

After a decades-long inconsistency between theory predictions and measurements, concerning in particular the polarizations, a major progress was brought by detailed LHC measurements of cross sections [2-9] and polarizations [1013], better calculations [14-20], and improved methods for unbiased data-theory comparisons [21-23].

Existing NLO SDC calculations can quantitatively describe the observed "universal unpolarized" scenario of LHC mid-rapidity data, where all states are seemingly produced with identical $p_{\mathrm{T}} / M$ distributions and zero polarizations [24]. From a conceptual point of view, however, the structure of the NRQCD factorization expansion, where three kinematically very different octet terms $\left({ }^{1} \mathrm{~S}_{0}^{[8]},{ }^{3} \mathrm{~S}_{1}^{[8]}\right.$, and ${ }^{3} \mathrm{P}_{J}^{[8]}$ ) dominate the production of $\mathrm{S}$-wave states and two singlet terms $\left({ }^{3} \mathrm{P}_{1}^{[1]}\right.$ and $\left.{ }^{3} \mathrm{P}_{2}^{[1]}\right)$ are additionally necessary (summed to the ${ }^{3} S_{1}^{[8]}$ term) to describe $\chi_{c 1, b 1}$ and $\chi_{c 2, b 2}$ production, does not seem to naturally match the exceptionally simple patterns seen in the experimental data. In fact, the theory accomplishes its formal success thanks to unexpected, precise cancellations.

While the seemingly superfluous complexity of the theory formulation is particularly apparent in the comparison with the remarkably simple experimental patterns, a certain degree of theory redundancy can be seen independently of any experimental observation. For S-wave quarkonium production, which is the focus of this paper, the dominating terms of the NRQCD expansion are the ${ }^{1} S_{0}^{[8]},{ }^{3} S_{1}^{[8]}$, and 


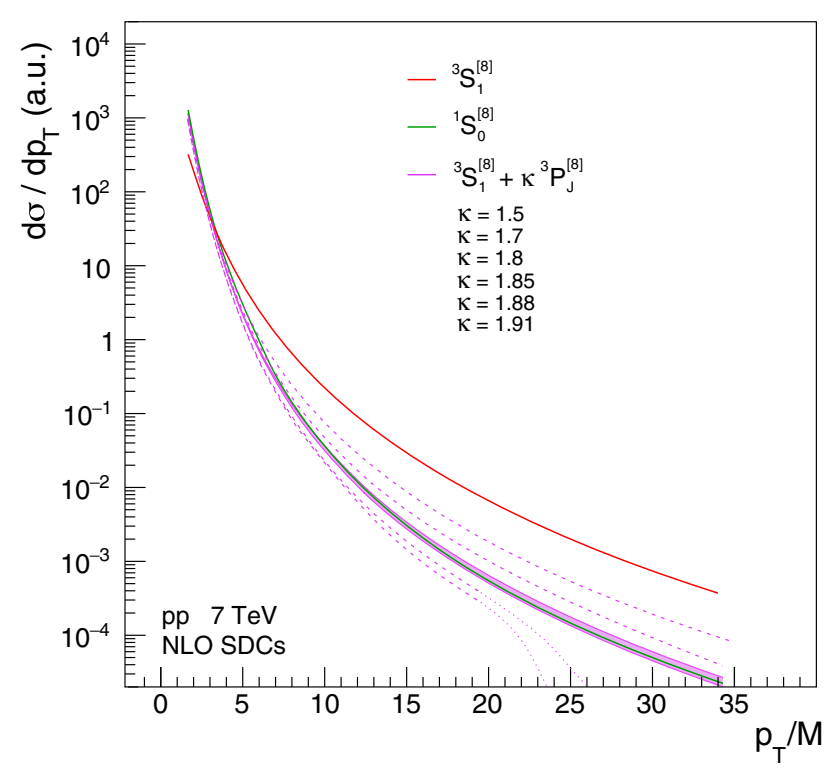

Fig. 1 The dominating colour-octet SDCs for the direct production of ${ }^{3} \mathrm{~S}_{1}$ quarkonia in NLO NRQCD, as functions of $p_{\mathrm{T}} / M:{ }^{1} \mathrm{~S}_{0}^{[8]}$ (green), ${ }^{3} S_{1}^{[8]}$ (red), and several ${ }^{3} S_{1}^{[8]}+\kappa{ }^{3} P_{J}^{[8]}$ combinations (magenta). The band represents the $\kappa=1.8-1.85$ range, which matches very well the shape of the (arbitrarily normalized) ${ }^{1} \mathrm{~S}_{0}^{[8]} \mathrm{SDC}$

${ }^{3} \mathrm{P}_{J}^{[8]}$ octets, all of the same order, $v^{4}$ (the ${ }^{3} \mathrm{~S}_{1}^{[1]}$ singlet term being negligible because of its small SDC). Throughout this paper we use the next-to-leading order (NLO) calculations of Refs. $[17,18]$ for the SDCs as functions of $p_{\mathrm{T}}$, in pp collisions at $\sqrt{s}=7 \mathrm{TeV}$ and mid rapidity.

Figure 1 shows that the shape of the ${ }^{1} \mathrm{~S}_{0}^{[8]} \mathrm{SDC}$ is indistinguishable from a linear combination of the two other terms, ${ }^{3} S_{1}^{[8]}+\kappa{ }^{3} \mathrm{P}_{J}^{[8]}$, with $\kappa$ between 1.8 and 1.85 . While in principle one would expect to fit experimental data with a superposition of three independent terms, leading to a definite determination of the three corresponding LDMEs, it turns out that the presently-available NLO SDCs are not independent kinematic templates and even very precise measurements of $p_{\mathrm{T}^{-}}$ differential cross sections will only be able to determine, in the best case, two parameters (as already noted in Ref. [15]). In other words, the NLO description of the $p_{\mathrm{T}}$ dependence of quarkonium production has three theory parameters, but only two degrees of freedom. This observation suggests the existence of a spurious element of complexity in the assumed base of subprocesses.

According to the present NLO knowledge of the SDCs, the polarization is a better discriminating observable. Figure 2 compares the calculated dilepton-decay polar anisotropy parameter, $\lambda_{\vartheta}$, as a function of $p_{\mathrm{T}} / M$, for the ${ }^{1} \mathrm{~S}_{0}^{[8]},{ }^{3} \mathrm{~S}_{1}^{[8]}$, and ${ }^{3} \mathrm{~S}_{1}^{[8]}+\kappa^{3} \mathrm{P}_{J}^{[8]}$ terms. Contrary to the case of the production yields, no value of $\kappa$ exists for which $\lambda_{\vartheta}\left({ }^{3} \mathrm{~S}_{1}^{[8]}+\kappa^{3} \mathrm{P}_{J}^{[8]}\right) \approx$ $\lambda_{\vartheta}\left({ }^{1} \mathrm{~S}_{0}^{[8]}\right)$ as a function of $p_{\mathrm{T}} / M$, and a momentumdependent polarization measurement should be able to dis-

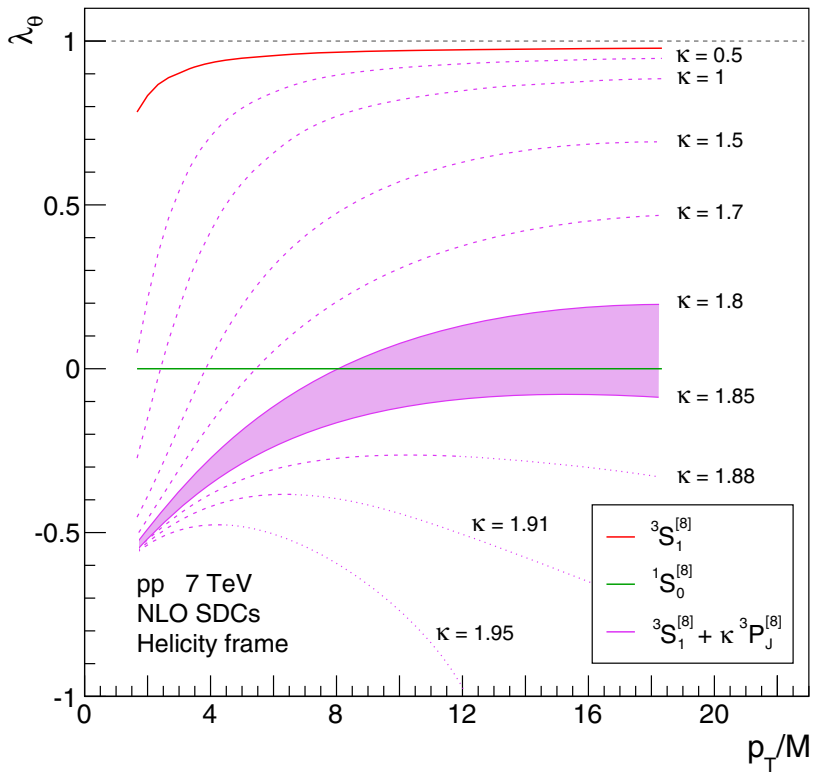

Fig. 2 The polarization parameter $\lambda_{\vartheta}$ for the different components of ${ }^{3} \mathrm{~S}_{1}$ quarkonium production in NLO NRQCD, as functions of $p_{\mathrm{T}} / M$ : ${ }^{1} \mathrm{~S}_{0}^{[8]}$ (green), ${ }^{3} \mathrm{~S}_{1}^{[8]}$ (red), and several ${ }^{3} \mathrm{~S}_{1}^{[8]}+\kappa{ }^{3} \mathrm{P}_{J}^{[8]}$ combinations (magenta). The band represents the $\kappa=1.8-1.85$ range, as in Fig. 1

entangle the three components. However, for $p_{\mathrm{T}} / M \gtrsim 10$, the ${ }^{3} S_{1}^{[8]}+\kappa{ }^{3} \mathrm{P}_{J}^{[8]}$ combination with $\kappa$ in the $1.8-1.85$ range becomes unpolarized, just as the ${ }^{1} \mathrm{~S}_{0}^{[8]}$ term. Therefore, at high $p_{\mathrm{T}}$, the polarization measurements are also unable to resolve more than two degrees of freedom in the space of the contributing LDMEs, at least for quarkonia produced at mid-rapidity in high-energy pp collisions.

It is worth noting that for $\kappa \gtrsim 1.85$ the ${ }^{3} \mathrm{~S}_{1}^{[8]}+\kappa{ }^{3} \mathrm{P}_{J}^{[8]}$ combination assumes unphysical behaviours, represented in Figs. 1 and 2 by the dotted lines: the $p_{\mathrm{T}} / M$ distribution shows seemingly anomalous changes of curvature at high $p_{\mathrm{T}} / M$ while the polarization parameter starts decreasing towards unphysical asymptotic values $\left(\lambda_{\vartheta}<-1\right)$. Intriguingly, the "degeneracy condition" $\kappa=1.8-1.85$ happens just before the border of the physical domain of positivity.

We will now see what the experimental measurements can add to the picture. Figures 3 and 4 show the mid-rapidity LHC measurements of cross sections and polarizations of different charmonium and bottomonium states.

As already mentioned, no significant differences are observed in the $p_{\mathrm{T}} / M$ dependences of yields and polarizations for the different states, despite their varying feeddown contributions (ranging between 0 and $40 \%$ ) from heavier quarkonia. This universal behaviour has been discussed in detail in Ref. [24], where it is shown that the $\chi_{c}$ feeddown contributions do not alter significantly, within the current experimental precision, the kinematic patterns of the directly-produced $\mathrm{J} / \psi$ mesons. It is, therefore, reasonable to compare the inclusive data (including feed-down) to the 


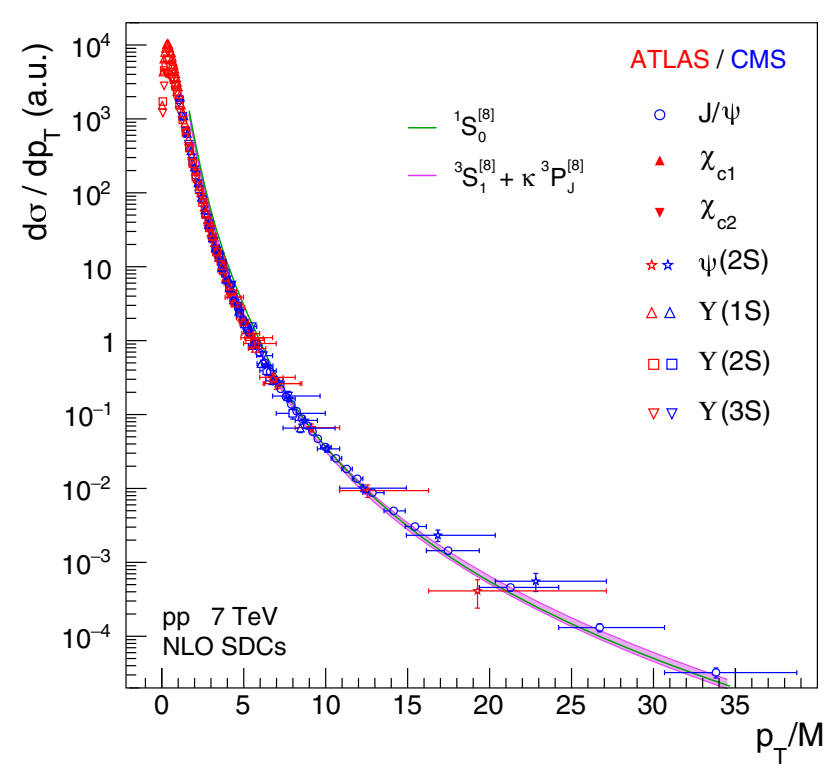

Fig. 3 Mid-rapidity quarkonium cross sections measured in pp collisions at $\sqrt{s}=7 \mathrm{TeV}$ by ATLAS (red markers) [3-5] and CMS (blue markers) [6,7], with normalizations arbitrarily adjusted to the $\mathrm{J} / \psi$ points to illustrate the universality of the $p_{\mathrm{T}} / M$ dependence. The data are compared to the shapes of the ${ }^{1} \mathrm{~S}_{0}^{[8]}$ octet (green) and of the ${ }^{3} \mathrm{~S}_{1}^{[8]}+\kappa{ }^{3} \mathrm{P}_{J}^{[8]}$ combination with $\kappa=1.8-1.85$ (magenta band)

NRQCD curves computed for direct production (excluding feed-down). For the curves themselves we use the NLO SDC calculations $[17,18]$ performed for a $Q \bar{Q}$ mass of $3 \mathrm{GeV}$, assuming the validity of the $p_{\mathrm{T}} / M$ scaling observed in cross section data as a general rule for individual processes. This approximation is justified by dimensional analysis reasonings applied to a single production mechanism [25], when $\sqrt{s}$ is large with respect to the momentum and mass of the observed state.

The figure shows that the scenario $\kappa=1.8-1.85$ is not just an hypothetical idea to be considered among many others but a scenario in rather good agreement with the presently existing quarkonium cross section and polarization measurements. For $p_{\mathrm{T}} / M \gtrsim 10$, the cross section measurements are compatible with the $p_{\mathrm{T}} / M$ distribution of the ${ }^{1} \mathrm{~S}_{0}^{[8]}$ term, only a small correction coming from other terms. The observed lack of polarization further supports the idea that the ${ }^{1} S_{0}^{[8]}$ term is close to reproducing the data with no need of further contributions. For high $p_{\mathrm{T}} / M$, the NRQCD expansion appears, therefore, as fully degenerate: all the data can be described by a single one of the basis terms, the ${ }^{1} S_{0}^{[8]}$ octet, or, equivalently, by the combination ${ }^{3} S_{1}^{[8]}+\kappa{ }^{3} \mathrm{P}_{J}^{[8]}$, with $\kappa=1.8-1.85$.

As previously mentioned, the degeneracy is broken for $p_{\mathrm{T}} / M<10$, where the NLO SDCs lead to different polarization predictions. However, the measured polarizations remain independent of $p_{\mathrm{T}} / M$ down to $p_{\mathrm{T}} / M \sim 1$. This

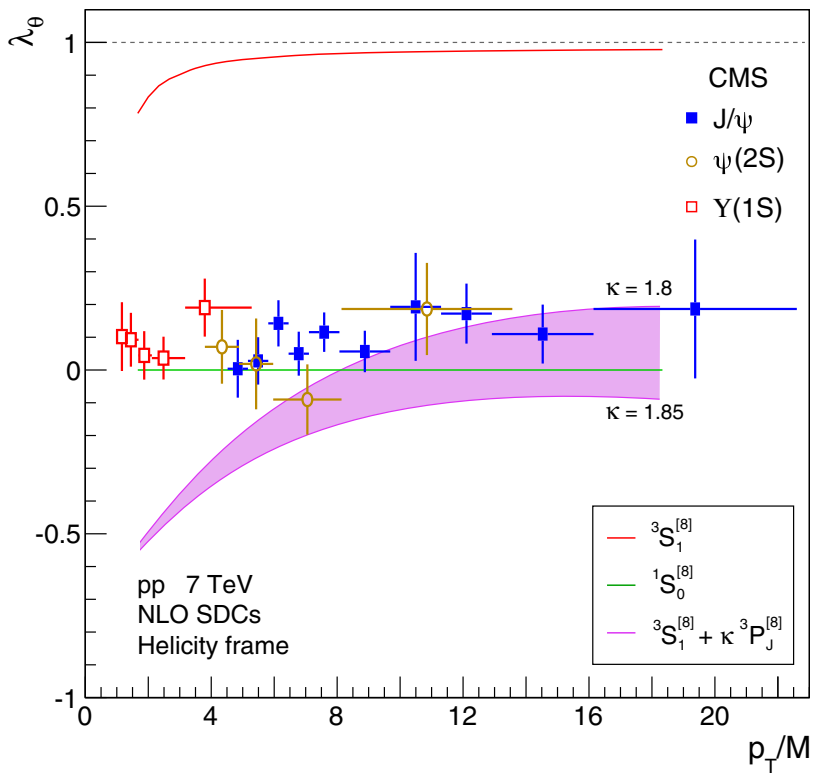

Fig. 4 The $\mathrm{J} / \psi, \psi(2 \mathrm{~S})$, and $\Upsilon(1 \mathrm{~S})$ polarization parameters as a function of $p_{\mathrm{T}} / M$, measured at mid-rapidity by the CMS experiment, compared to the NRQCD calculations for the ${ }^{1} \mathrm{~S}_{0}^{[8]}$ (green) and ${ }^{3} \mathrm{~S}_{1}^{[8]}$ (red) octets, as well as to the ${ }^{3} \mathrm{~S}_{1}^{[8]}+\kappa^{3} \mathrm{P}_{J}^{[8]}$ combination with $\kappa=1.8-1.85$ (magenta band)

means that the comparison between the measured data and the NLO SDCs clearly excludes a significant contribution of the ${ }^{3} S_{1}^{[8]}+\kappa{ }^{3} \mathrm{P}_{J}^{[8]}$ term, which is expected to be either strongly $p_{\mathrm{T}}$ dependent or strongly polarized, as shown in Fig. 2. Even a relatively small ${ }^{3} S_{1}^{[8]}+\kappa^{3} \mathrm{P}_{J}^{[8]}$ contribution to the quarkonium production yields, of around $10 \%$, is already excluded by the presently existing polarization data, independently of the value of $\kappa$. In other words, the mid-rapidity quarkonium polarization measurements made at the LHC indicate a strong hierarchy of cross section contributions, clearly enhancing the ${ }^{1} \mathrm{~S}_{0}^{[8]}$ octet and suppressing the individual ${ }^{3} \mathrm{~S}_{1}^{[8]}$ and ${ }^{3} \mathrm{P}_{J}^{[8]}$ terms, not only their partially cancelling combination. This observation constitutes a significant violation of the $v$-scaling rules, according to which the three LDMEs should have the same order of magnitude.

Such a strong and unexpected constraint relies on the accuracy of the present NLO calculations. Uncertainties from the factorization scale and from the quark masses, mainly affecting the SDC normalizations, have a negligible impact on our considerations, which are entirely based on shape differences. Since the shapes of the NLO ${ }^{1} S_{0}^{[8]}$ and ${ }^{3} S_{1}^{[8]}$ SDCs are similar to the leading order (LO) versions, it is reasonable to expect that future computations of higher-order contributions will not reveal significant changes, as indicated by the already known partial corrections due to fragmentation contributions $[19,20]$. The ${ }^{3} \mathrm{P}_{J}^{[8]}$ term, instead, shows drastic changes from LO to NLO, including a change of sign, and the fragmentation corrections are large $[19,20]$. One may 
argue, hence, that higher-order corrections could change the $p_{\mathrm{T}} / M$ dependence of the ${ }^{3} \mathrm{~S}_{1}^{[8]}+\kappa^{3} \mathrm{P}_{J}^{[8]}$ polarization, especially towards low $p_{\mathrm{T}} / M$. To reach a situation where the polarization measurements would no longer exclude this contribution, so that the $v^{2}$ hierarchy predicted by NRQCD $\left({ }^{1} \mathrm{~S}_{0}^{[8]}\right.$ $\sim{ }^{3} S_{1}^{[8]} \sim{ }^{3} \mathrm{P}_{J}^{[8]}$ ) would be recovered, those future higherorder corrections should necessarily lead to a flatter dependence of $\lambda_{\vartheta}$ on $p_{\mathrm{T}} / M$. The presently available quarkonium polarization measurements are already sufficiently precise to suggest that those future computations need to reach a suspiciously high level of fine tuning, but it is clear that new polarization measurements, of significantly improved precision, are needed to provide the ultimate constraint on the NRQCD hierarchy puzzle.

In particular, high-precision measurements confirming beyond doubt the scenario of vanishing and $p_{\mathrm{T}}$-independent polarization would leave zero margin for a ${ }^{3} \mathrm{~S}_{1}^{[8]}+\kappa^{3} \mathrm{P}_{J}^{[8]}$ contribution other than unpolarized and $p_{\mathrm{T}}$-independent. The crucial importance of such measurements can be appreciated by considering the two possible conclusions: (a) if the calculated ${ }^{3} \mathrm{~S}_{1}^{[8]}+\kappa{ }^{3} \mathrm{P}_{J}^{[8]}$ term remains significantly different from unpolarized and shows some level of $p_{\mathrm{T}}$ dependence (as is the case of the NLO computations), then we will be forced to conclude that its contribution is zero and quarkonium production is driven entirely by the ${ }^{1} \mathrm{~S}_{0}^{[8]}$ channel; (b) if improved (higher order) calculations lead to $\lambda_{\vartheta}\left({ }^{3} S_{1}^{[8]}+\kappa^{3} \mathrm{P}_{J}^{[8]}\right) \sim 0$, the validity of the NRQCD $v$-scaling rules will be formally rescued, but the three octet terms would collapse to a single one, given that they would then have identical observable kinematic properties. This outcome would clearly point to the existence of a more natural and fundamental formulation of the factorization expansion, reflecting less degrees of freedom, valid at least in the conditions of mid-rapidity quarkonium production in high-energy pp collisions.

The best way to accurately probe the low- $p_{\mathrm{T}} / M$ region is to use $\Upsilon(\mathrm{nS})$ measurements, ideally with well-resolved $1 \mathrm{~S}, 2 \mathrm{~S}$ and $3 \mathrm{~S}$ states to indirectly examine possible effects from the feed-down decay contributions of the P-wave states. Accessing the high- $p_{\mathrm{T}} / M$ kinematical domain (easier to study with $\mathrm{J} / \psi$ and $\psi(2 \mathrm{~S})$ data, given the lower masses and higher production cross sections) will be crucial to probe if quarkonium production is completely dominated by a single unpolarized production mechanism (the ${ }^{1} \mathrm{~S}_{0}^{[8]}$ octet term, say) or if there are two (or more) polarized terms that seemingly cancel each other in the existing data. High-precision measurements revealing no polarization, independently of $p_{\mathrm{T}}$, could no longer be explained with coincidental cancellations between cross section terms: if the present hints of cancellations result from a conspiracy between the low experimental precision and the limited kinematical domain covered by data, a $p_{\mathrm{T}}$-dependent residual polarization should become visible at sufficiently high $p_{\mathrm{T}}$. It should be easy to vastly reduce the statistical uncertainties of the presently available mid-rapidity LHC quarkonium polarization measurements, which are exclusively based on the $7 \mathrm{TeV}$ data collected by CMS in 2011, corresponding to an integrated luminosity of only $5 \mathrm{fb}^{-1}$, much less than the $140 \mathrm{fb}^{-1}$ collected at $13 \mathrm{TeV}$ between 2016 and 2018. Polarization measurements reported by the LHCb collaboration $[12,13,26]$ will also be very valuable to precisely investigate the low $p_{\mathrm{T}}$ region, once the $p_{\mathrm{T}} / M$ scaling studies [24] will have been extended to the forward rapidity range covered by that experiment. The systematic uncertainties, likely to become the dominating ones in a large range of the measured $p_{\mathrm{T}}$ spectrum, can be reduced by performing the measurements in at least two (orthogonal) polarization frames and by also reporting measurements of the $\lambda_{\varphi}$ and $\lambda_{\vartheta \varphi}$ azimuthal anisotropy parameters [21]. Measuring the $\tilde{\lambda}$ frame-invariant parameter [27] in several polarization frames will also help uncovering potential biases.

It is important to note that the experimental conditions of the LHC data considered in this paper may represent our best chance of exploring the hierarchy and possible limit degeneracies in the NRQCD expansion. Indeed, given the very high collision energies, we can access mid-rapidity data up to relatively high $p_{\mathrm{T}} / M$ values with a good statistical precision, so that we can study a phase space window where quarkonium production is dominated by $2 \rightarrow 2$ processes of the kind $g g \rightarrow Q \bar{Q}+g$. In this configuration the $Q \bar{Q}$ pre-resonance can "freely" assume all $J$ values up to, in principle, $J=3$ (with $S=0$ or $S=1$ ); there is, in principle, no a priori topological constraint removing some of these terms, nor imposing a specific $J_{z}$ (i.e., a polarization). Instead, data obtained in experimental conditions dominated by specific individual channels would necessary be affected by additional constraints, as in the case of singlet-driven production via virtual photon in $e^{+} e^{-}$collisions, or in kinematic domains where $2 \rightarrow 1$ production becomes important and the $Q \bar{Q}$ directly inherits the angular momentum state of the system of colliding partons. In short, it could well be that only data collected in optimal conditions, where no special angular momentum constraints are directly imposed on the produced $Q \bar{Q}$, allow us to probe the existence of an "unbroken degeneracy" at a fundamental level.

In summary, we have shown the existence of a kinematic domain in high-energy pp collisions, $p_{\mathrm{T}} / M \gtrsim 10$ at midrapidity, where the description of quarkonium production using the current formulation of a $v^{2}$ expansion in ${ }^{2 S+1} L_{J}$ Fock-states, together with NLO SDC calculations, becomes degenerate. Indeed, the observed lack of polarization and universal $p_{\mathrm{T}} / M$ dependence of the yields can be described, indifferently, by one of the expansion terms alone, the ${ }^{1} \mathrm{~S}_{0}^{[8]}$ octet, or by a combination of the other two, the ${ }^{3} S_{1}^{[8]}+\kappa{ }^{3} \mathrm{P}_{J}^{[8]}$ linear combination (and, obviously, by a mixture of the two cases, combined with unobservable proportions). This 
degeneracy is broken for $p_{\mathrm{T}} / M<10$ by the polarization prediction, because the ${ }^{3} \mathrm{~S}_{1}^{[8]}+\kappa{ }^{3} \mathrm{P}_{J}^{[8]}$ term acquires a strong $p_{\mathrm{T}} / M$ dependence, differentiating it from the constant and unpolarized ${ }^{1} \mathrm{~S}_{0}^{[8]}$ octet. However, such change of regime is not at all observed in the existing measurements, which seamlessly prolong their unpolarized behaviour down to the lowest data point, at $p_{\mathrm{T}} / M \sim 2$. This implies a strong violation of the $v$-scaling rules and an unexpected dominance of the ${ }^{1} \mathrm{~S}_{0}^{[8]}$ term. Future high-precision quarkonium polarization measurements have the potential to rule out the current formulation of the factorization expansion beyond any possible recovery, if they confirm the unpolarized scenario suggested by the currently available data. Fine-tuned higher-order corrections could provide SDC calculations that would resuscitate the ${ }^{3} S_{1}^{[8]}+\kappa{ }^{3} \mathrm{P}_{J}^{[8]}$ term, but at the expense of turning it, necessarily, into another unpolarized term, thereby exposing a full degeneracy of the currently postulated Fock-space expansion. Such an outcome, rather than solving the riddle, would raise an even more compelling question: does a more essential and natural description of the experimental observations exist? The search for explanations of why and how the degeneracy appears in the considered phase space (midrapidity and/or high $p_{\mathrm{T}} / M$, in high-energy pp collisions), will certainly bring advances in the fundamental understanding of quarkonium production.

We would like to thank Hua-Sheng Shao, who kindly provided tables of the NLO calculations of the SDCs.

Data Availability Statement This manuscript has no associated data or the data will not be deposited. [Authors' comment: Data sharing not applicable to this article as no datasets were generated or analysed during the current study.]

Open Access This article is distributed under the terms of the Creative Commons Attribution 4.0 International License (http://creativecomm ons.org/licenses/by/4.0/), which permits unrestricted use, distribution, and reproduction in any medium, provided you give appropriate credit to the original author(s) and the source, provide a link to the Creative Commons license, and indicate if changes were made. Funded by SCOAP $^{3}$.

\section{References}

1. G.T. Bodwin, E. Braaten, P. Lepage, Phys. Rev. D 51, 1125 (1995). https://doi.org/10.1103/PhysRevD.55.5853, https://doi. org/10.1103/PhysRevD.51.1125. arXiv:hep-ph/9407339. [Erratum: Phys. Rev. D55,5853(1997)]
2. ATLAS Coll., Eur. Phys. J. C 76, 283 (2016). https://doi.org/10. 1140/epjc/s10052-016-4050-8, arXiv:1512.03657

3. ATLAS Coll., JHEP. 09, 079 (2014). https://doi.org/10.1007/ JHEP09(2014)079, arXiv:1407.5532

4. ATLAS Coll., JHEP. 07, 154 (2014). https://doi.org/10.1007/ JHEP07(2014)154, arXiv:1404.7035

5. ATLAS Coll., Phys. Rev. D 87, 052004 (2013). https://doi.org/10. 1103/PhysRevD.87.052004, arXiv: 1211.7255

6. C.M.S. Coll., Phys. Rev. Lett. 114, 191802 (2015). https://doi.org/ 10.1103/PhysRevLett.114.191802. arXiv:1502.04155

7. C.M.S. Coll., Phys. Lett. B 749, 14 (2015). https://doi.org/10.1016/ j.physletb.2015.07.037. arXiv:1501.07750

8. LHCb Coll., Eur. Phys. J. C 71, 1645 (2011). https://doi.org/10. 1140/epjc/s10052-011-1645-y, arXiv:1103.0423

9. LHCb Coll., Eur. Phys. J. C 72, 2100 (2012). https://doi.org/10. 1140/epjc/s10052-012-2100-4, arXiv:1204.1258

10. C.M.S. Coll., Phys. Lett. B 727, 381 (2013). https://doi.org/10. 1016/j.physletb.2013.10.055. arXiv: 1307.6070

11. C.M.S. Coll., Phys. Rev. Lett. 110, 081802 (2013). https://doi.org/ 10.1103/PhysRevLett.110.081802. arXiv: 1209.2922

12. LHCb Coll., Eur. Phys. J. C 73, 2631 (2013). https://doi.org/10. 1140/epjc/s10052-013-2631-3, arXiv:1307.6379

13. LHCb Coll., Eur. Phys. J. C 74, 2872 (2014). https://doi.org/10. 1140/epjc/s10052-014-2872-9, arXiv:1403.1339

14. M. Butenschön, B. Kniehl, Mod. Phys. Lett. A 28, 1350027 (2013). https://doi.org/10.1142/S0217732313500272, arXiv:1212.2037

15. Y.-Q. Ma, K. Wang, K.-T. Chao, Phys. Rev. D 84, 114001 (2011). https://doi.org/10.1103/PhysRevD.84.114001, arXiv:1012.1030

16. K.-T. Chao et al., Phys. Rev. Lett. 108, 242004 (2012). https://doi. org/10.1103/PhysRevLett.108.242004. arXiv:1201.2675

17. H.-S. Shao, Y.-Q. Ma, K. Wang, K.-T. Chao, Phys. Rev. Lett 112, 182003 (2014). https://doi.org/10.1103/PhysRevLett.112.182003. arXiv: 1402.2913

18. H.-S. Shao, Comput. Phys. Commun. 198, 238 (2016). https://doi. org/10.1016/j.cpc.2015.09.011. arXiv:1507.03435

19. G.T. Bodwin, H.S. Chung, U.-R. Kim, J. Lee, Phys. Rev. Lett. 113, 022001 (2014). https://doi.org/10.1103/PhysRevLett.113.022001. arXiv: 1403.3612

20. G.T. Bodwin et al., Phys. Rev. D 93, 034041 (2016). https://doi. org/10.1103/PhysRevD.93.034041. arXiv:1509.07904

21. P. Faccioli, C. Lourenço, J. Seixas, H. Wöhri, Eur. Phys. J. C 69, 657 (2010). https://doi.org/10.1140/epjc/s10052-010-1420-5. arXiv: 1006.2738

22. P. Faccioli et al., Phys. Lett. B 736, 98 (2014). https://doi.org/10. 1016/j.physletb.2014.07.006. arXiv:1403.3970

23. P. Faccioli et al., Phys. Lett. B 773, 476 (2017). https://doi.org/10. 1016/j.physletb.2017.09.006. arXiv:1702.04208

24. P. Faccioli et al., Eur. Phys. J. C 78, 268 (2018). https://doi.org/10. 1140/epjc/s10052-018-5755-7. arXiv:1802.01106

25. P. Faccioli, C. Lourenço, M. Araújo, J. Seixas, Eur. Phys. J. C 78, 118 (2018). https://doi.org/10.1140/epjc/s10052-018-5610-x. arXiv: 1802.01102

26. LHCb Coll., JHEP 12, 110 (2017). https://doi.org/10.1007/ JHEP12(2017)110, arXiv:1709.01301

27. P. Faccioli, C. Lourenço, J. Seixas, Phys. Rev. D 81, 111502 (2010). https://doi.org/10.1103/PhysRevD.81.111502. arXiv:1005.2855 